\title{
Removal of Hydrophobic Organic Contaminants from Aqueous Solutions by Sorption onto Biodegradable Polyesters
}

\author{
Yukiko Matsuzawa ${ }^{1}$, Zen-Ichiro Kimura ${ }^{1}$, Yoshiro Nishimura ${ }^{2}$, \\ Mikio Shibayama ${ }^{2}$, Akira Hiraishi ${ }^{1^{*}}$ \\ ${ }^{1}$ Department of Ecological Engineering, Toyohashi University of Technology, Toyohashi, Japan \\ ${ }^{2}$ Mikawa Textile Research Institute, Aichi Prefecture, Gamagori, Japan \\ E-mail: hiraishi@eco.tut.ac.jp \\ Received November 7, 2009; revised November 24, 2009; accepted January 21, 2010
}

\begin{abstract}
Sorption by biodegradable polyesters of several aromatic chemicals as model compounds for hydrophobic organic contaminants (HOCs) was studied. The biodegradable polyesters used were poly(butylene succinate), poly(3-hydroxybutyrate-co-3-hydroxyvalerate), poly( $\varepsilon$-caprolactone), poly(butylene succinate/ terephthalate), and poly(L-lactic acid). Petrochemical plastics, low-density polyethylene and polyethylene terephtalate, were used for comparison. The target HOCs were biphenyl, bisphenol-A, dibenzofuran, diethylstilbestrol, nonylphenol, and chlorophenols. The treatment of the HOC solutions with each of the biodegradable polyesters resulted in the nearly complete removal of the chemicals by sorption from the aqueous phase, except for the case of poly(L-lactic acid). Low-density polyethylene adsorbed biphenyl and dibenzofuran selectively, and polyethylene terephtalate did not adsorb any of the HOCs. The adsorptive interaction between the plastics and the HOCs might be related to both the glass transition temperature of the former and the nature of the latter as defined by the Fujita's inorganicity/organicity ratio. The toxic effect of 3,5-dichlorophenol on bacterial growth in liquid culture was removed by the addition of a biodegradable polyester. These results provide a basis for the applicability of the biodegradable plastics as the adsorbents for the removal of HOCs from aquatic environments.
\end{abstract}

Keywords: Aromatic Chemicals, Hydrophobic Organic Contaminants, Sorption, Biodegradable Polyester

\section{Introduction}

A wide variety of aromatic chemicals released from anthropogenic sources into the environment are recognized as harmful and persistent contaminants. Such hydrophobic organic contaminants (HOCs) are concentrated in biosystems through the food chain, thereby bringing about a potential threat of biotic environments [1]. Therefore, the problem of how to detoxify and remove HOCs in the environment is central to research in environmental science and technology. One of the most widely used approaches to this subject is the removal of HOCs using appropriate sorbents such as activated carbon, siliceous materials, zeolite, and synthetic resins [2]. In recent years, biopolymers and petrochemical plastics as the sorbents of HOCs have gained more attention. It has been shown that aromatic compounds are adsorbed by soil organic matter and biopolymers such as lignin, chitin, chitosan, and cellulose [2-5]. Plastic debris dispersed as persistent pollutants in marine ecosystems [6] have been reported to act as transporters of HOCs $[7,8]$. Aromatic compounds in foods and liquid media are adsorbed by polyethylene [9-11]. The sorption of aromatic compounds on polyethylene as a membrane material was studied [12]. HOCs from municipal wastewater treatment plant effluents could be removed by sorption onto polypropylene [13]. Although the mechanism of adsorptive interaction between plastics and HOCs has not yet been fully elucidated, the available information suggests that sorption technology using these polymers has great promise as a tool for HOC removal from the environment.

Biodegradable polyesters, which have attracted 
much attention as alternatives of conventional plastic packages have physicochemical properties resembling petrochemical plastics $[14,15]$. It is therefore of special interest to investigate whether biodegradable polyesters as well as petrochemical plastics adsorb HOCs. To date, there has been no fundamental information about the adsorptive interaction between biodegradable polyesters and HOCs. In this study, we report that selected biodegradable polyesters are able to adsorb aromatic chemicals more effectively than selected petrochemical plastics. We discuss the applicability of biodegradable plastics as potent adsorbents for the removal of HOCs from aquatic environments. To our knowledge, this report is the first to demonstrate that biodegradable plastics are able to adsorb HOCs.

\section{Materials and Methods}

\subsection{Chemicals}

The aromatic chemicals used as model compounds for HOCs were biphenyl (BP), bisphenol-A (BPA), dibenzofuran (DF), diethylstilbestrol (DES), and nonylphenol (NP). Also, phenol and its chlorinated derivatives, 2monochlorophenol (2-CP) and 3,5-dichlorophenol (3,5-DCP) were used. Among these chemicals, BPA, DES, and NP are suspected of being endocrine disruptors. Some congeners of chlorophenols are known to have inhibitory effects as uncouplers on energy metabolism in organisms [16,17]. All these chemicals were purchased from Wako Pure Chemical Industries (Osaka, Japan). The physicochemical characteristics of the HOCs as defined by the Fujita's inorganicity $(i)$ and organicity $(o)$ characters, which are based on the concept of van der Waals interaction and electronic affinity, respectively [18, 19], were determined using an Excel Macro program available at a web site (http://www.ecosci.jp/sheet/ orgs_help.html).

\subsection{Biodegradable Polyesters}

Commercially available biodegradable polyesters were used as the sorbents. These polymer samples were poly( $\varepsilon$-caprolactone) (PCL) (Daicel Chemical Industries, Tokyo, Japan), poly(butylene succinate/terephthalate) (Ecoflex, EF) (BASF Japan Ltd., Tokyo, Japan), poly(butylene-succinate) (PBS) (Showa Highpolymer Co., Hyogo, Japan), poly(3-hydroxybutyrate-co-8\%-3hydroxyvalerate) (PHBV) (Japan Monsanto, Tokyo, Japan), and poly(L-lactic acid) (PLA) (Mitsui Chemicals, Inc., Tokyo, Japan). For comparison, low-density polyethylene (LDPE) (Mitsui Chemicals) and polyethylene terephtalate (PET) were used as representatives of conventional petrochemicals. For testing, fibers of the plas- tics were prepared by the melt-spinning method. The melt spinning machine was a $35 \mathrm{~mm}$ single screw extruder $(\mathrm{L} / \mathrm{D}=28)$ provided with a spinning nozzle having 6 holes with a diameter of $1.0 \mathrm{~mm}$. The melt extrusion temperature was $200{ }^{\circ} \mathrm{C}$ for all samples. The fibers were extruded into a cooling water bath at $45^{\circ} \mathrm{C}$ for PLA and $20{ }^{\circ} \mathrm{C}$ for the others; the output was about $6 \mathrm{~g} \mathrm{~min}^{-1}$. The take-up speed for the fiber was $7 \mathrm{~m} \mathrm{~min}^{-1}$. The diameter of the fiber was set at $1 \mathrm{~mm}$ in all cases. The plastic fibers thus prepared were cut into an appropriate size before use.

\subsection{Characterization of Biodegradable Polyesters}

Melting temperature was measured with a Rigaku TAS100 differential scanning calorimeter heated at $10{ }^{\circ} \mathrm{C}$ $\min ^{-1}$ under a nitrogen gas flow. Wide-angle X-ray diffraction measurement of polymer samples was performed using the Shimadzu XD-D1 system. Cu-Ko radiation $(\lambda=0.1542 \mathrm{~nm})$ was used as the light source. The degree of crystallization ( $X c^{\text {Xray }}$ ) was calculated according to the following equation: $X c^{\text {Xray }}(\%)=100 \times I_{\text {crystal }}$ $/\left(I_{\text {crystal }}+I_{\text {amorphous }}\right)$, where $I_{\text {crystal }}$ is the area of the crystalline peak and $I_{\text {crystal }}+I_{\text {amorphous }}$ is the overall area. The physicochemical characteristics of the biodegradable polyesters and petrochemical plastics used are shown in Table 1.

\subsection{Spectrophotometric Monitoring of HOC Removal}

Saturated aqueous solutions of HOCs in $10 \mathrm{mM}$ phosphate buffer ( $\mathrm{pH}$ 7.0) were prepared. Before use, the saturated solutions were diluted to have an ultraviolet absorbance of 0.2 to 0.4 at $248 \mathrm{~nm}$ for BP, $280 \mathrm{~nm}$ for $\mathrm{DF}, 245 \mathrm{~nm}$ for DES, and $276 \mathrm{~nm}$ for chlorophenols. Polymer fibers measuring $10 \mathrm{~mm}$ in length $(0.5 \mathrm{~g}$ each) were introduced into $10 \mathrm{ml}$ screw-capped glass vials containing $5 \mathrm{ml}$ of a diluted HOC solution and shaken on a reciprocal shaker $\left(140\right.$ strokes $\left.\mathrm{min}^{-1}\right)$ for $24 \mathrm{~h}$ at ambient temperature $\left(25^{\circ} \mathrm{C}\right)$. Then, an aliquot of the solution was sampled and analyzed spectrophotometrically with a Shimadzu BioSpec 1600 spectrophotometer.

\subsection{HPLC Monitoring of HOC Removal}

HOC solutions at a concentration of $0.15-0.35 \mathrm{mM}$ were prepared and treated for $24 \mathrm{~h}$ with polymer fibers as noted above. An aliquot of the aqueous solution was sampled at an appropriate interval during the $24-\mathrm{h}$ period of treatment and directly analyzed by reverse-phase HPLC or extracted with ethylacetate prior to the HPLC analysis. The solvent extraction was made twice in total of $5 \mathrm{ml}$. HPLC was performed with a Shimadzu model LC10A liquid chromatograph equipped with a Zorbax 
ODS column (4.6 [i.d.] × $250 \mathrm{~mm}$ ) and a Shimadzu SPD-2A photodiode array detector. BP and DF were eluted with $90 \%$ methanol at a flow rate of $1.0 \mathrm{ml} \mathrm{min}^{-1}$ and monitored at 248 and $280 \mathrm{~nm}$, respectively. BPA, $\mathrm{NP}$, and chlorophenols were eluted with 70-80\% methanol at a flow rate of $0.5 \mathrm{ml} \mathrm{min} \mathrm{min}^{-1}$ and monitored at $276 \mathrm{~nm}$. DES was eluted with $70 \%$ methanol at a flow rate of $1.0 \mathrm{ml} \mathrm{min}-1$ and monitored at $245 \mathrm{~nm}$. The concentration of HOCs was determined by comparing HPLC peak areas of samples with those of the standards at known concentrations.

\subsection{Recovery of Adsorbed HOCs}

After $24 \mathrm{~h}$ of treatment as noted above, the polymer fibers adsorbing HOCs were collected from the aqueous phase and washed three times with pure water. The washed loss was less than the detectable limit. Then, the HOCs on the polymer were extracted twice with ethylacetate in total of $5 \mathrm{ml}$ and analyzed directly by reverse-phase HPLC as noted above.

\subsection{Temperature Dependency}

The effect of temperature on the interaction between PLA and DF was studied. PLA fibers were cut to give a length of $5 \mathrm{~mm}$ and immersed in $5 \mathrm{ml}$ of DF solution (2 $\left.\mu \mathrm{g} \mathrm{ml}{ }^{-1}\right)$ in glass tubes. The test tubes were kept at ambient temperature $\left(25^{\circ} \mathrm{C}\right)$ and in a water bath at $50{ }^{\circ} \mathrm{C}$ and $75^{\circ} \mathrm{C}$. At appropriate intervals of incubation, DF was extracted from the solution in the test tubes and analyzed by HPLC as described above.

\subsection{Bacterial Growth with 3,5-DCP and PHBV}

Escherichia coli strain IAM 12119 (the type strain) and Bacillus subtilis IAM 12118 (the type strain), both of which were obtained from the IAM Culture Collection Center, the University of Tokyo (Tokyo, Japan) were used. The test organisms were pre-grown aerobically in PBY liquid medium [20]. A $50 \mu \mathrm{m}$ aliquot of cultures at the late exponential phase of growth was introduced into four screw-capped test tubes (30-ml capacity) containing $6 \mathrm{ml}$ of PBY medium. A small amount of a filtersterilized 3,5-DCP solution was added to one of the test tubes at the beginning to give a concentration of $27 \mu \mathrm{g}$ $\mathrm{ml}^{-1}$ and incubated aerobically at $28{ }^{\circ} \mathrm{C}$ on a reciprocal shaker (designated test 1). In tests 2 and 3, the 3,5-DCP solution was added to the culture after $3 \mathrm{~h}$ of incubation. In test 3 , autoclaved PHBV fibers $\left(50 \mathrm{mg} \mathrm{ml}^{-1}\right)$ were further added aseptically to the cultures after $6 \mathrm{~h}$ of incubation. The control culture was made without any additive. Growth was monitored by measuring the optical density at $660 \mathrm{~nm}\left(\mathrm{OD}_{660}\right)$ using an Amar
Table 1. Biodegradable polyesters and petrochemical polymers used.

\begin{tabular}{cccc}
\hline Polyester & $\begin{array}{c}\text { Crystallinity } \\
(\%)\end{array}$ & $\begin{array}{c}\text { Melting tem- } \\
\text { perature }\left({ }^{\circ} \mathrm{C}\right)\end{array}$ & $\begin{array}{c}\text { Glass tran- } \\
\text { sition tem- } \\
\text { perature } \\
\left({ }^{\circ} \mathrm{C}\right)\end{array}$ \\
\hline Biodegradable & & & \\
EF & 21.3 & 115 & -30 \\
PBS & 44.5 & 113 & -32 \\
PCL & 58.8 & 59 & -60 \\
PHBV & 52.6 & 158 & 4 \\
PLA & 20.9 & 163 & 60 \\
Non-biodegrad- & & & \\
able & & & \\
LDPE & 45.8 & 120 & -120 \\
PET & $\mathrm{nd}^{\mathrm{a}}$ & 260 & 70 \\
\hline
\end{tabular}

${ }^{\mathrm{a}}$ nd, not determined.

sham-Pharmacia Novaspec spectrophotometer. Cellmorphology were checked by phase-contrast microscopy and epifluorescence microscopy with SYBR Green I staining [21]. Viability of cells was estimated by the tetrazolium reduction method using 5-cyano-2,3-ditoryl tetrazolium chloride (CTC) at single cell resolution [22]. The concentration of 3,5-DCP remaining in the liquid phase was monitored by HPLC as noted above.

\section{Results and Discussion}

\subsection{Removal Efficiency with Different Polyesters}

The aqueous solutions of different aromatic compounds as model HOCs were shaken in the presence or absence of a biodegradable polyester added as the sorbent. Then, the removal of HOCs from the aqueous phase after $24 \mathrm{~h}$ of treatment was checked spectrophotometrically. As examples, UV absorption spectra of BP, BPA, DF, and DES solutions with or without PCL applied are shown in Figure 1. These aromatic solutions showed their characteristic absorption maxima between 200 and $300 \mathrm{~nm}$ in the absence of PCL (Figures 1(a)-1(d), traces I). On the other hand, the addition of PCL to the solutions resulted in the disappearance of absorption peaks in all cases (Figures 1(a)-1(d), traces II), indicating that the HOCs were removed from the aqueous phase by sorption onto PCL. Similar effects on the aromatic solutions were observed with the addition of EF, PBS, and PHBV, whereas the addition of PLA to the solutions exerted little or no spectral change under the experimental conditions (data not shown).

HPLC experiments were performed to quantitatively estimate the efficiency of HOC removal by different biodegradable polyesters as the adsorbents compared LDPE and PET. All of the biodegradable polyesters used, except PLA, adsorbed all test HOCs effectively at a removal efficiency of more than $98 \%$ within $24 \mathrm{~h}$ of treatment (Table 2). Much lower efficiency of HOC removal 


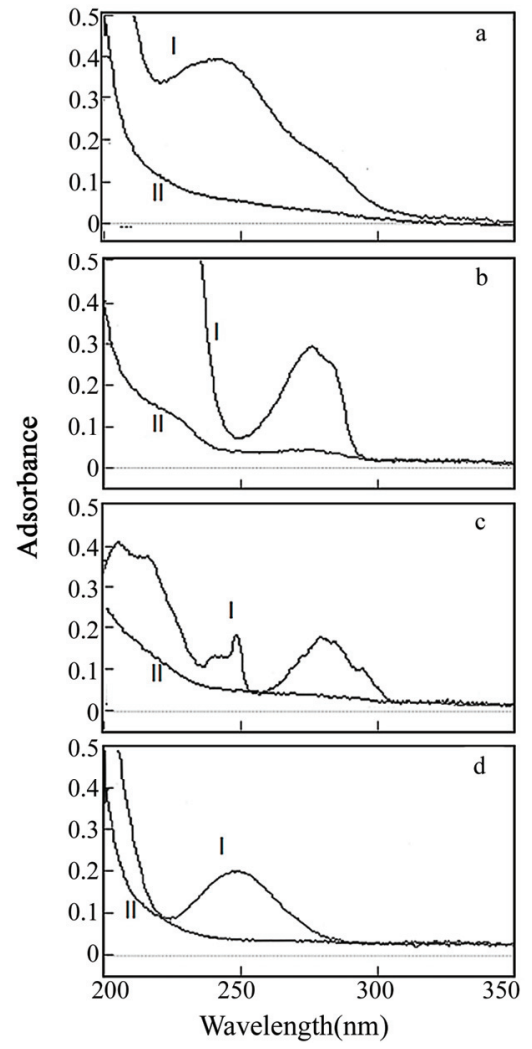

Figure 1. Absorption spectra of aqueous solutions of BP (a), BPA (b), DF (c), and DES (d) with and without PCL applied. (I) spectra without PCL applied; (II) spectra after 24 h treatment with PCL.

$(\leq 22 \%)$ was obtained with PLA and PET. The HOC removal patterns of LDPE were different in some degree from those with the biodegradable polyesters. Namely, LDPE adsorbed BP and DF effectively but removed much less amounts of BPA and DES from the solutions.

The adsorption behavior of phenol, 2-CP, and 3,5DCP on the biodegradable polyesters was analyzed in greater detail to study whether the sorption efficiency is related to the number of chlorine substituents, i.e., hydrophobicity of the chemicals. As example, the time course of the removal of phenol and the chlorophenols by PCL is shown in Figure 2. The removal efficiency became high with increasing numbers of chlorine substituents on phenol; the adsorbed amounts of phenol, 2-CP, and 3,5-DCP after $24 \mathrm{~h}$ of treatment were 20, 50, and $75 \mu \mathrm{mol} \mathrm{g}{ }^{-1}$, respectively. These results suggest that the hydrophobic interaction between the polymers and HOCs is an important factor affecting the sorption.

PBS and PHBV had similar effects on the chlorophenols but exhibited lower sorption efficiencies than PCL. The adsorbed amounts of the chlorophenols after $24 \mathrm{~h}$ of treatment with PBS and PHBV were 70-95\% and 50$80 \%$ of those with PCL, respectively, whereas PLA did not adsorb the chlorophenols.
Table 2. Removal of aromatic compounds by sorption onto different biodegradable and non-biodegradable polyesters.

\begin{tabular}{cccccc}
\hline $\begin{array}{c}\text { Polyester } \\
\text { used }\end{array}$ & BP & BPA & DF & DES & NP \\
\hline $\begin{array}{c}\text { Biodegrad- } \\
\text { able }\end{array}$ & & & & & \\
\hline EF & $>99$ & $>99$ & $>99$ & $>99$ & $>99$ \\
PBS & $>99$ & $98 \pm 1$ & $>99$ & $99 \pm 1$ & $>99$ \\
PCL & $>99$ & $>99$ & $>99$ & $>99$ & $>99$ \\
PHBV & $>99$ & $98 \pm 1$ & $>99$ & $98 \pm 1$ & $98 \pm 1$ \\
PLA & $18 \pm 2$ & $22 \pm 2$ & $11 \pm 1$ & $13 \pm 1$ & $\mathrm{nt}^{\mathrm{b}}$ \\
\hline Non-biode- & & & & & \\
gradable & & & & & \\
\hline LDPE & $97 \pm 2$ & $9 \pm 4$ & $>99$ & $27 \pm 1$ & $\mathrm{nt}$ \\
PET & $8 \pm 2$ & $8 \pm 1$ & $6 \pm 1$ & $9 \pm 1$ & $\mathrm{nt}$ \\
\hline
\end{tabular}

a \% Removal after $24 \mathrm{~h}$ of treatment; the initial concentration of aromatics ranged from 0.15 to $0.30 \mu \mathrm{mol}(23-68 \mu \mathrm{g}) \mathrm{ml}^{-1}$. ${ }^{\mathrm{b}} \mathrm{nt}$, not tested.

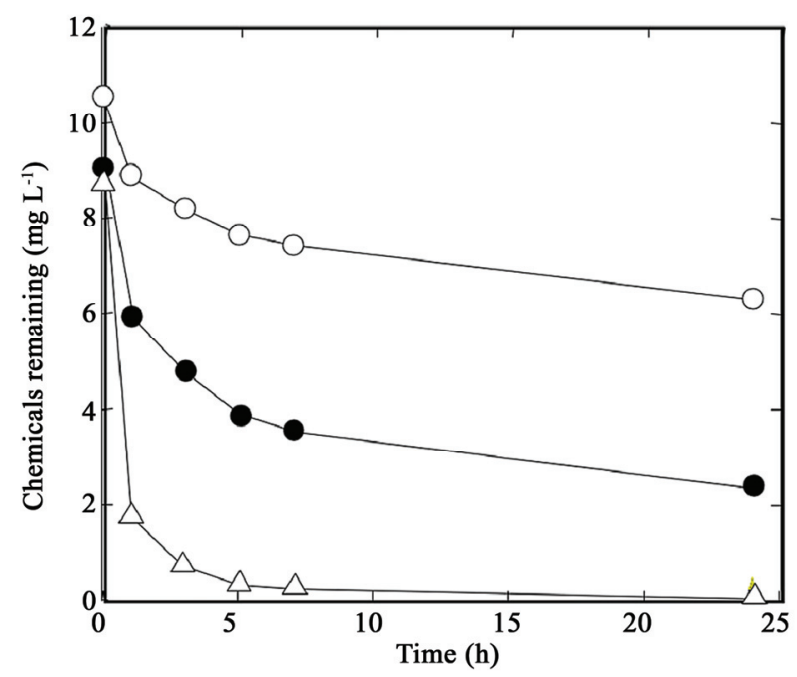

Figure 2. Removal of phenol (open circles), 2-CP (solid circles), and 3,5-DCP (triangles) from the aqueous solutions by sorption onto PCL.

\subsection{HOC Removal Efficiency with Different Amounts of Adsorbents}

Removal efficiency of 2-CP with different amounts of PCL added as an adsorbent was further studied under the same conditions as described for Figure 2. As shown in Figure 3, the ratio of the concentration of 2-CP in the liquid phase equilibrated after $24 \mathrm{~h}$ of treatment $\left(C_{\mathrm{e}}\right)$ to its initial concentration $\left(C_{0}\right)$ decreased linearly with increasing amounts of PCL as the absorbent. Preliminary experiments also showed that a much faster decrease in the $C_{\mathrm{e}} / C_{0}$ ratio was observed with increasing amounts of a granular type of PCL or PHBV (unpublished data). These results suggest that HOC removal efficiency depends upon the surface area of biodegradable polyesters as the absorbents. 


\subsection{Recovery of Adsorbed HOCs from Polymers}

If the hydrophobic interaction is mainly involved in the sorption of HOCs onto the surface of polymer, the compounds absorbed should be fully recoverable by extraction with an organic solvent. To examine this, the BPA and DF solutions were treated with EF, PBS, PCL, and $\mathrm{PHBV}$, and the net amounts of the chemicals extracted separately from the solution and the polymers were determined. As shown in Table 3, the total amount of BPA and DF recovered from each of the 4 polyesters accounted for only 19 to $56 \%$ of the initial amount in the respective solutions. These results suggest that the mechanism of HOC sorption by the polymers is more than a simple hydrophobic interaction.

\subsection{Effects of Temperature on Sorption}

In view of the results noted above, the affinity between the polymers and HOCs may be related to the chemical nature of the former compounds as well as of the latter. A possible determinant of this is grass transition temperature. PCL, PBS, EF, and PHBV have a lower grass transition temperature ranging from $-60{ }^{\circ} \mathrm{C}$ to $4{ }^{\circ} \mathrm{C}$ (see Table 1). As noted above, these polymers absorbed the HOCs effectively at room temperature, and the removal efficiency of the chlorophenols decreased in the following order: PCL (glass transition temperature, $-60{ }^{\circ} \mathrm{C}$ ) $>$ PBS $\left(-32^{\circ} \mathrm{C}\right)>\operatorname{PHBV}\left(4^{\circ} \mathrm{C}\right)$. On the other hand, PLA, having a much higher glass transition temperature $(60$ ${ }^{\circ} \mathrm{C}$ ), did not adsorb the chemicals effectively at room temperature. PET, whose grass transition temperature is $70^{\circ} \mathrm{C}$, showed similar behavior to PLA.

To determine the effect of temperature on the interaction of chemicals with polymers, a DF solution was treated with PLA at $25{ }^{\circ} \mathrm{C}, 50{ }^{\circ} \mathrm{C}$, and $75{ }^{\circ} \mathrm{C}$, and the chemical in the hot solution was extracted with ethylacetate. As shown in Figure 4, more than $90 \%$ of DF in the hot solution at $75{ }^{\circ} \mathrm{C}$ was removed by PLA, contrasting well with the control test with the solution at $25^{\circ} \mathrm{C}$ in which DF was hardly removable. The removal efficiency at $50{ }^{\circ} \mathrm{C}$, which is lower than the glass transition temperature of PLA, was also low.

Based on the aforementioned results, it may be logical to conclude that the glass transition temperature of polymers is another important determinant of their affinity to HOCs. The mobility of the amorphous phase of plastics much increases at a higher temperature than glass transition temperature, and this physical state is possibly critical for elevating in the HOC-sorption efficiency. In this context, it is of special interest to further study the relationship between the sorption efficiency and cristallinity of the plastics. Although LDPE has a low glass transition temperature $\left(-120^{\circ} \mathrm{C}\right)$, it did not adsorb some of the tested HOCs (i.e., BPA and DES) effectively. At this time, we are unable to find a plausible reason for this with certainty but can assume

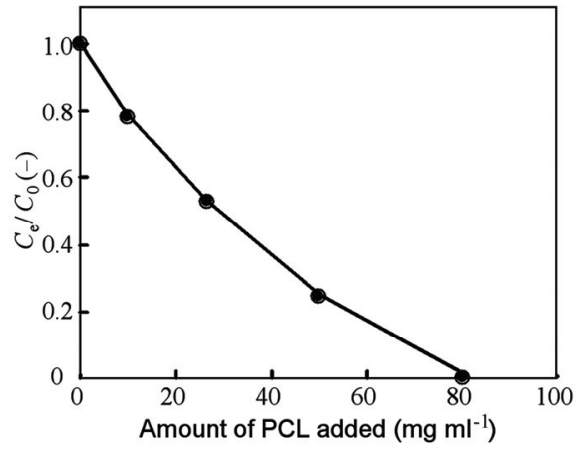

Figure 3. Removal efficiency of 2-CP as a function of the amount of PCL added as an adsorbent. $C_{\mathrm{e}}$, equilibrated concentration of 2-CP; $C_{0}$, initial concentration of 2-CP.

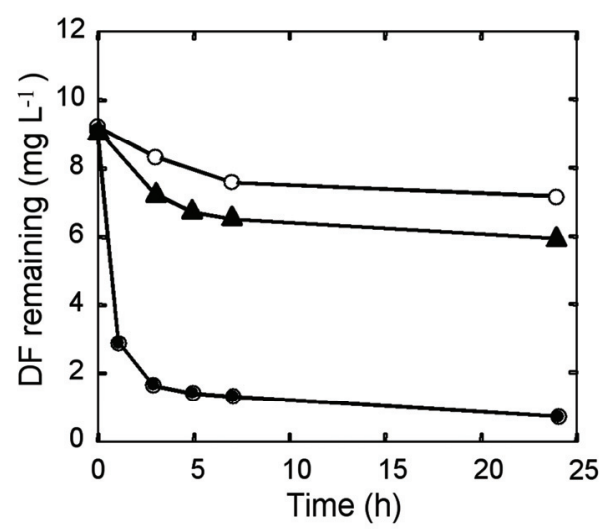

Figure 4. Removal of DF from the aqueous solution at $25^{\circ} \mathrm{C}$ (open circles), $50^{\circ} \mathrm{C}$ (triangles), and $75^{\circ} \mathrm{C}$ (closed circles) by sorption onto PLA.

Table 3. Recovery of adsorbed aromatics from biodegradable polyesters by extracting with ethylacetate.

\begin{tabular}{ccc}
\hline \multirow{2}{*}{ Polyester } & \multicolumn{2}{c}{$\%$ Recovery of: } \\
& BPA & DF \\
\hline EF & $34 \pm 6$ & $22 \pm 4$ \\
PBS & $18 \pm 4$ & $19 \pm 6$ \\
PCL & $21 \pm 5$ & $31 \pm 6$ \\
PHBV & $55 \pm 8$ & $56 \pm 9$ \\
\hline
\end{tabular}

that the interactions between HOCs and the plastics possibly depend upon the physicochemical nature of the former as well as of the latter.

\subsection{Significance of $i / o$ Characters of Chemicals in Sorption}

For more comprehensive understanding of the adsorptive behavior of HOCs, we considered the Fujita's $i / o$ characters, which are the parameters based on the concept of van der Waals interaction and electronic affinity, respectively. The $i / o$ ratio is useful to predict the physicochemical characteristics of chemical compounds in the 
liquid phase $[18,19]$. It can be used as a criterion of adsorbability as well as of solubility [23]. As shown in Figure 5, the $i$ values for BP and DF are much lower than those for BPA and DES. In addition, BP and DF have a lower $i / o$ ratio than BPA and DES. Thus, one can assume that chemicals having a low $i$ value and a low $i / o$ ratio, such as $\mathrm{BP}$ and $\mathrm{DF}$, are adsorbed strongly by hydrophobic polymers in one fashion, while BPA and DES, having a high $i$ value, interact with them in another manner. This characteristic of chemicals is an additional important factor to consider their sorption behavior onto the biodegradable polyesters as well as onto petrochemical plastics. Abe et al. [23] have used the $i / o$ value to explain the adsorptive mechanism on activated carbon in the liquid phase.

\subsection{Elimination of Toxic Effects of 3,5-DCP by Sorption}

A wide variety of HOCs such as 3,5-DCP have toxic effects on growth and viability of organisms. Therefore, it is important to elucidate whether these effects can be reduced by sorption using biodegradable polyesters. To know about this, two phylogenetically different bacteria were tested for growth in the presence of 3,5-DCP and PHBV. The tested organisms were Bacillus subtilis and Escherichia coli, both of which are unable to degrade PHBV and the other polyesters used in this study. The results of the experiment with Bacillus subtilis are shown in Figure 6. In tests 1 and 2, the addition of 3,5-DCP into the culture resulted in the complete inhibition of bacterial growth. In test 3, on the other hand, the addition of PHBV into the 3,5-DCP-containing cultures resulted in the restoration of its growth (Figure 6(a), triangles) along with the removal of 3,5-DCP from the aqueous phase (Figure 6(b)). Epifluorescence microscopy showed that there were no differences in cell morphology and CTC-reducing activity at single cell resolution between the test 3 culture (Figure 6(a), triangles) and the control culture (Figure 6(a), solid diamonds) at the stationary phase of growth. Similar effects of PHBV were obtained with 3,5-DCP-containing cultures of Escherichia coli (data not shown).

As reported above, our attempt to eliminate the toxic effect of 3,5-DCP on bacteria by using PHBV as the adsorbent has given positive results. These results suggest the usefulness of sorption technology using biodegradable polyesters in protecting aquatic organisms from environmental hazards with HOCs. Our concurrent study has shown that the biodegradable polyesters are able to adsorb several other harmful aromatic compounds such as benzene, toluene, ethylbenzene, and xylene, and, in this respect, they are superior to activated carbon (unpublished observations). Further study should give more

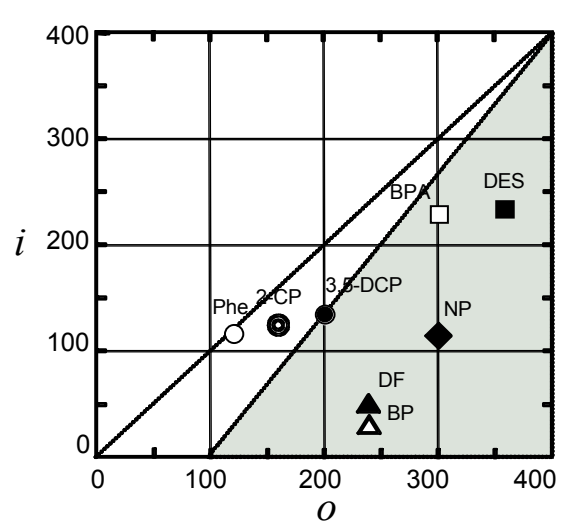

Figure 5. Fujita's $i / o$ characters of HOCs used in this study. Symbols: open circle, phenol (Phe); double circle, 2monochlorophenol (2-CP); solid circle, 3,5- dichlorophenol (3,5-DCP); open triangle, biphenyl (BP); open square, bis-phenol A (BPA); solid square, diethylstilbestrol (DES); solid triangle, dibenzofuran (DF); solid diamond, nonylphenol (NP). The compounds in the shaded area may be those adsorbed effectively by the biodegradable polyesters.
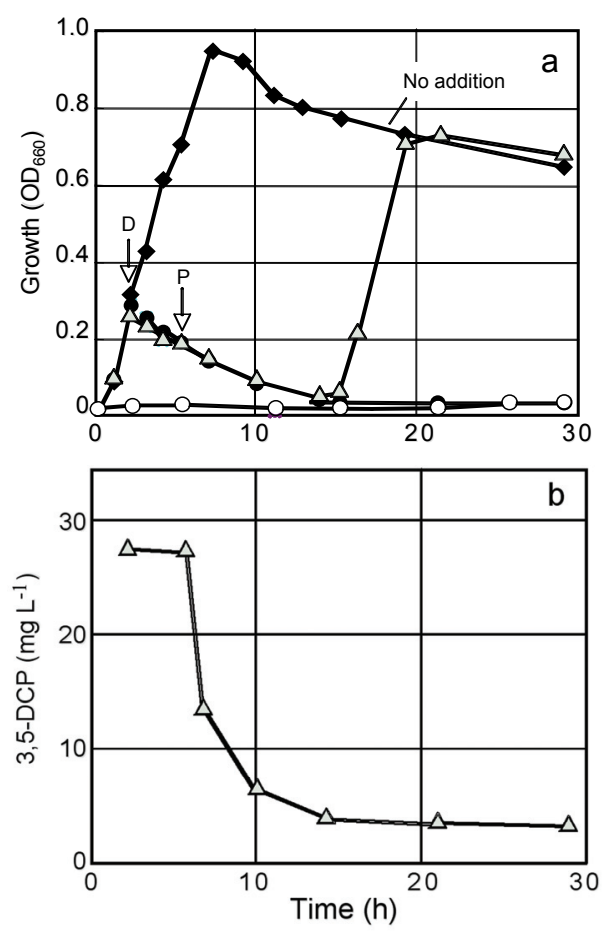

Figure 6. Effects of 3,5-DCP and PHBV on growth of Bacillus subtilis strain IAM 12118 (a) and change in the concentration of 3,5-DCP in the culture supernatant in test 3 (b). Symbols in Figure 4(a): diamonds, growth without 3,5-DCP (control); open circles, growth with 3,5-DCP (test 1); closed circles, growth with 3,5-DCP added after $3 \mathrm{~h}$ of incubation (shown by an arrow with $D$ ) (test 2); triangles, growth with 3,5-DCP and PHBV added after $3 \mathrm{~h}$ (shown by an arrow with $D$ ) and $5 \mathrm{~h}$ (shown by an arrow with $P$ ) of incubation, respectively (test 3 ). 
definitive information about the applicability of the biodegradable polyesters as novel adsorbents in sorption technology for the environmental remediation of $\mathrm{HOCs}$.

\section{Conclusions}

The present study was designed to evaluate the applicability of the biodegradable polyesters, i.e., EF, PBS, PCL, PHBV, and PLA, as the sorbents for the removal of HOCs from the environment. The results of this study have demonstrated that all of the biodegradable polyesters tested, except PLA, are able to adsorb the aromatic compounds as model HOCs in aqueous solutions at room temperature. In the HOCs removal efficiency, the biodegradable polyesters are superior to conventional petrochemical plastics such as LDPE and PET. Although the sorption mechanism for this has not yet been fully understood, it is likely that both the physicochemical characteristics of the plastics (e.g., glass transition temperature) and the nature of the HOCs as defined by the Fujita's $i / o$-characters is involved. The toxic effect of $3,5-\mathrm{DCP}$ on microbial growth in liquid cultures can be eliminated by sorption onto biodegradable polyesters.

\section{Acknowledgments}

We are grateful to Prof. H. Tsuji, Department of Ecological Engineering, Toyohashi University of Technology, for critical comments and stimulating discussions. This study was supported in part by grant K1544 from the Ministry of the Environment, Japan, and by a grant-in-aid for Scientific Research (No. 17310046) from the Ministry of Education, Culture, Sports, Science and Technology, Japan.

\section{References}

[1] G. Ross, "The public health implications of polychlorinated biphenyls (PCBs) in the environment," Ecotoxicology and Environmental Safety, Vol. 59, No. 3, pp. 275-291, 2004.

[2] M. Ahmaruzzaman, "Adsorption of phenolic compounds on low-cost adsorbents: A review," Advances in Colloid and Interface Science, Vol. 143, No. 1-2, pp. 48-67, 2008.

[3] A. S. Gunasekara and B. Xing, "Sorption and desorption of naphthalene by soil organic matter: Importance of aromatic and aliphatic components," Journal of Environmental Quality, Vol. 32, No. 1, pp. 240-246, 2003.

[4] X. Wang, R. Cook, S. Tao, and B. Xing, "Sorption of organic contaminants by biopolymers: Role of polarity, structure and domain spatial arrangement," Chemosphere, Vol. 66, No. 8, pp. 1476-1484, 2007.

[5] X. Wang, K. Yang, S. Tao, and B. Xing, "Sorption of aromatic organic contaminants by biopolymers: Effects of $\mathrm{pH}$, copper (II) complexation, and cellulose coating," Environmental Science \& Technology, Vol. 41, No. 1, pp. 185-191, 2007.

[6] J. G. Derraik, "The pollution of the marine environment by plastic debris: A review," Marine Pollution Bulletin, Vol. 44, No. 9, pp. 842-852, 2002.

[7] S. Endo, R. Takizawa, K. Okuda, H. Takada, K. Chiba, H. Kanehiro, H. Ogi, R. Yamashita, and T. Date, "Concentration of polychlorinated biphenyls (PCBs) in beached resin pellets: Variability among individual particles and regional differences," Marine Pollution Bulletin, Vol. 50, No. 10, pp. 1103-1114, 2005.

[8] Y. Mato, T. Isobe, H. Takada, H. Kanehiro, C. Ohtake, and T. Kaminuma, "Plastic resin pellets as a transport medium for toxic chemicals in the marine environment," Environmental Science \& Technology, Vol. 35, No. 2, pp. 318-324, 2001.

[9] J. Chen and S. Chen, "Removal of polycyclic aromatic hydrocarbons by low density polyethylene from liquid model and roasted meat," Food Chemistry, Vol. 90, No. 3, pp. 461-469, 2004.

[10] P. Simko, "Factors affecting elimination of polycyclic aromatic hydrocarbons from smoked meat foods and liquid smoke flavorings," Molecular Nutrition \& Food Research, Vol. 49, No. 7, pp. 637-647, 2005.

[11] P. Simko, P. Simon, and V. Khunova, "Removal of polycyclic aromatic hydrocarbons from water by migration into polyethylene," Food Chemistry, Vol. 64, No. 2, pp. 157-161, 1999.

[12] Y. Kiso, T. Kitao, and K. Nishimura, “Adsorption properties of aromatic compounds on polyethylene as a membrane material," Journal of Applied Polymer Science, Vol. 74, No. 5, pp. 1037-1043, 1999.

[13] V. S. Muhandiki, Y. Shimizu, Y. A. Adou, and S. Matsui, "Removal of hydrophobic micro-organic pollutants from municipal wastewater treatment plant effluents by sorption onto synthetic polymeric adsorbents: Batch sorption experiments," Environmental Technology, Vol. 28, No. 3, pp. 415-424, 2007.

[14] R. Auras, B. Harte, and S. Selke, "An overview of polylactides as packaging materials," Macromolecular Bioscience, Vol. 4, No. 9, pp. 835-864, 2004.

[15] J. M. Luengo, B. García, A. Sandoval, G. Naharro, and E. R. Olivera, "Bioplastics from microorganisms," Current Opinion in Microbiology, Vol. 6, No. 3, pp. 251-260, 2003.

[16] A. Hiraishi and T. Kawagishi, "Effects of chemical uncouplers on microbial biomass production, metabolic activity, and community structure in an activated sludge system," Microbes \& Environments, Vol. 17, No. 4, pp. 197-204, 2002.

[17] F. X. Ye, D. S. Shen, and Y. Li, "Reduction in excess sludge production by addition of chemical uncouplers in activated sludge batch cultures," Journal of Applied Microbiology, Vol. 95, No. 4, pp. 781-786, 2003.

[18] H. Inoue, A. Uehara, and M. Nango, "Separation method for organic compounds (in Japanese)," Shokabo, Tokyo, 1990. 
[19] S. Saito, A. Tanoue, and M. Matsuo, "The i/o-characters to evaluate $n$-octanol/water partition coefficient in bioconcentration of organic chemicals in fish," Chemosphere, Vol. 24, No. 1, pp. 89-95, 1992.

[20] H. Futamata, T. Uchida, N. Yoshida, Y. Yonemitsu, and A. Hiraishi, "Distribution of dibenzofuran-degrading bacterial in soils polluted with different levels of polychlorinated dioxins," Microbes \& Environments, Vol. 19, No. 2, pp. 172-177, 2004.

[21] S. T. Khan, Y. Horiba, N. Takahashi, and A. Hiraishi, "Activity and community composition of denitrifying bacteria in poly(3-hydroxybutyrate-co-3-hydroxy-valerate)-using solid-phase denitrification processes," Mi- crobes \& Environments, Vol. 22, No. 1, pp. 20-31, 2007.

[22] Y. Fujii and A. Hiraishi, "Combined use of cyanoditolyl tetrazolium staining and flow cytometry for detection of metabolically active bacteria in a fed-batch composting process," Microbes \& Environments, Vol. 24, No. 1, pp. 57-63, 2009.

[23] I. Abe, K. Hayashi, M. Kitagawa, and Y. Urahata, "Adsorptive mechanism on activated carbon in the liquid phase. III. The relationship between the physical constants of organic compounds and their adsorbabilities on activated carbon from an aqueous solution," Bulletin of the Chemical Society of Japan, Vol. 53, No. 5, pp. 1199-1205, 1980. 